\title{
Organisationsgesellschaft neu gedacht: Vom Archetyp zu neuen Formen der Organisation
}

\author{
Nadine Arnold • Raimund Hasse (iD · Hannah Mormann
}

Eingegangen: 5. Februar 2021 / Angenommen: 24. August 2021 / Online publiziert: 30. September 2021 (C) Der/die Autor(en) 2021

Zusammenfassung Während die Organisationssoziologie vornehmlich auf die Analyse von Organisationen und zwischenorganisatorischen Arrangements konzentriert ist, finden wir in verschiedenen anderen Teilgebieten der Soziologie die Einschätzung, dass die Rolle von Organisationen hinsichtlich ihrer gesellschaftlichen Effekte oftmals nur unzureichend reflektiert wird. Dieser Beitrag zielt darauf ab, konzeptionelle Voraussetzungen zur Überwindung dieser Situation zu schaffen. In Abschn. 2 wird auf das Konzept der Organisationsgesellschaft rekurriert, so wie es in der zweiten Hälfte des 20. Jahrhunderts entwickelt worden ist. Als gemeinsames Problem dieser Forschungsbeiträge wird identifiziert, dass sie sich auf eine bestimmte - hier als Archetyp bezeichnete - Organisationsform beziehen, deren Dominanz im 21. Jahrhundert fragwürdig geworden ist. In Abschn. 3 wird deshalb vorgeschlagen, neue Organisationsformen einzubeziehen. Insbesondere wird auf HyperOrganisation, partielle Organisation und Plattform-Organisation eingegangen. Es werden zwei Schwachpunkte dieser Konzepte identifiziert: Erstens suggerieren die entsprechenden Beiträge eine Ersetzung des Archetyps und vernachlässigen zudem andere neuartige Organisationsformen; zweitens beziehen sie gesellschaftliche Voraussetzungen und Folgen nur selektiv ein. Abschließend werden Perspektiven zur Überwindung dieser gravierenden Schwachstellen diskutiert.

\footnotetext{
N. Arnold $\cdot$ R. Hasse $(\bowtie) \cdot$ H. Mormann Soziologisches Seminar, Universität Luzern Frohburgstr. 3, 6002 Luzern, Schweiz

E-Mail: raimund.hasse@unilu.ch

N. Arnold

E-Mail: nadine.arnold@unilu.ch

H. Mormann

E-Mail: hannah.mormann@unilu.ch
} 
Schlüsselwörter Bürokratie · Hyper-Organisation · Partielle Organisation · Plattform-Organisation · Gesellschaftliche Entwicklung

\title{
Organizational Society Revisited: from Archetype to New Forms of Organization
}

\begin{abstract}
Whereas organizational sociology mainly focuses on the analysis of organizations and interorganizational arrangements, in various other subfields of sociology we find the opinion that the role of organizations regarding effects on society is often insufficiently reflected. This article aims to address conceptual prerequisites for overcoming this situation. In section 2 the concept of organizational society is referred back to, as has been developed in the second half of the twentieth century. As a common problem of these research contributions, it has been identified that they refer to a certain organizational form-here labelled as archetype-whose dominance in the twenty-first century has become questionable. Therefore, in section 3 it is suggested that new organizational forms should be considered. In particular, hyper-organization, partial organization, and platform organization are discussed. Two weaknesses of these concepts are identified: firstly, the appropriate contributions suggest an integral replacement of the archetype and also neglect other novel organizational forms; secondly, they only selectively include social prerequisites and sequelae. Finally, perspectives to overcome these major weaknesses are discussed.
\end{abstract}

Keywords Bureaucracy · Hyper-organization · Partial organization · Platform organization $\cdot$ Societal development

\section{Einleitung}

Der Elefant im Raum ist eine hilfreiche Metapher, um den aktuellen Stand der Forschung zum Verhältnis von Organisation und Gesellschaft zu verbildlichen. Sie bezeichnet ein Problem, das für alle offensichtlich ist, aber dennoch nicht angesprochen wird. ${ }^{1}$ Im vorliegenden Fall bringt sie auf den Punkt, dass Organisationen wie Unternehmen, Parteien und Nonprofits zwar oft eine große, wenn nicht gar fundamentale Rolle in vielen Bereichen der Gesellschaft spielen, diese umfassende organisatorische Durchdringung in soziologischen Analysen jedoch nur unzureichend thematisiert wird. Der vorliegende Beitrag zielt darauf ab, diese Situation zu ändern und Fragen der Organisation in das Zentrum soziologischer, aber eben nicht unbedingt nur organisationssoziologischer Analysen zu rücken.

Im Kern handelt es sich um eine Forschungsperspektive, die unter dem Stichwort Organisationsgesellschaft zusammengefasst worden ist (Gabriel 1979). Durch die besondere Aufmerksamkeit gegenüber neuen Formen der Organisation sollen im Folgenden notwendige Bedingungen zur Identifikation von Konturen der Organi-

\footnotetext{
1 Die Metapher ist weder zu verwechseln mit dem Elefanten im Porzellanladen (der auf ein ungeschicktes, unbeholfenes Verhalten hinweisen würde), noch damit, aus einer Mücke einen Elefanten zu machen (was dafür steht, etwas viel bedeutsamer zu machen als es ist).
} 
sationsgesellschaft im 21. Jahrhundert geschaffen werden. Ziel ist jedoch nicht die Ausarbeitung eines detaillierten Forschungsprogramms, sondern die Sensibilisierung für den oftmals vernachlässigten Zusammenhang von Organisation und Gesellschaft. Ausdrücklich angemerkt sei, dass mit der Begriffsverwendung Organisationsgesellschaft keinesfalls suggeriert werden soll, die moderne Gesellschaft oder Teilbereiche wie Wirtschaft, Wissenschaft und Politik ließen sich als bloßer Organisationszusammenhang beschreiben oder gar wie eine einzige Organisation betreiben, sodass Organisationstheorie als Ersatz für Makrosoziologie fungieren könnte (kritisch hierzu bereits Kühl 2015).

Ausgangspunkt der Forschungsperspektive Organisationsgesellschaft ist die lapidare Beobachtung, dass Organisationen in der Gesellschaft eine gravierende Rolle spielen, und zwar praktisch überall. Die Untersuchung dieser organisatorischen Durchdringung und ihrer gesellschaftlichen Relevanz ist im deutschsprachigen Raum überwiegend an systemtheoretische und neo-institutionalistische Herangehensweisen geknüpft worden (Hasse 2003; Jäger und Schimank 2005; Schimank 2010; Kühl 2015). Es gibt jedoch ein viel breiteres Spektrum an Theorieschulen, die ebenso Anknüpfungspunkte bieten, weil sie Organisationen oder bestimmte Organisationsformen für die gesellschaftliche Entwicklung als zentral erachtet haben; man denke etwa an totale Institutionen bei Goffman (1995), an den Zusammenhang von kognitiven und organisatorischen Strukturen der Wissenschaft bei Whitley (1984), an den von Foucault aufgezeigten Stellenwert von Strafanstalten und psychiatrischen Kliniken (z. B. Foucault 1996) oder an Organisationsfragen im Zusammenhang mit gesellschaftlichen Konflikten (Dahrendorf 1957). Allerdings handelt es sich um Ansätze mit einem bestimmten theoriegeschichtlichen und gesellschaftlichen Hintergrund, sodass jeweils zu prüfen ist, inwiefern diese Ansätze in das 21. Jahrhundert übertragen werden können.

Sucht man deshalb nach aktuellen Anknüpfungspunkten, dann sind es bemerkenswerterweise Forscher aus einem breiteren Spektrum soziologischer Teilgebiete, also nicht unbedingt aus der Organisationsforschung und auch nicht aus der Allgemeinen Soziologie, welche darauf aufmerksam machen, dass die Bedeutung von Organisationen vielfach übersehen oder unterschätzt wird. Während McQuarrie und Marwell (2009) in der Stadtsoziologie eine ,fehlende organisatorische Dimension“ feststellen, nutzt beispielsweise die Bewegungsforschung den Begriff der „NGOization“, um den oft unbemerkten Einfluss von Nichtregierungsorganisationen auf soziale Bewegungen und in der karitativen Hilfe sichtbar zu machen (z. B. Borchgrevink 2017; Paternotte 2016). In der Ungleichheitsforschung ist die bislang unbeachtete Rolle von Organisationen von Tomaskovic-Devey und Avent-Holt (2019) herausgearbeitet worden. Auf ähnliche Weise geben Agrarsoziologen zu bedenken, dass die gewählte Organisationsform für die Ausgestaltung der landwirtschaftlichen Produktion einen Unterschied machen kann (Groot Kormelinck et al. 2019). Organisationen werden maßgeblich dafür verantwortlich gemacht, ob und inwiefern umwelt- und sozialverträgliche Ernährungssysteme gesellschaftlich bestehen können (Duncan und Pascucci 2017). Neue Einsichten zur Rolle der Organisation für die Gesellschaft liefern schließlich auch wirtschaftssoziologische Untersuchungen, etwa zum Einfluss von Finanzexperten und Investoren (Jung und Dobbin 2014) sowie dem Downsizing von Unternehmen im Zuge von Finanzkrisen (Fligstein und Shin 2007). Ebenso wer- 
den Schlüsselunternehmen der digitalen Ökonomie wie Alphabet, Amazon, Apple und Facebook (Dolata 2015) in der Digitalisierungsforschung als Schrittmacher der gesellschaftlich folgenreichen Produkt- und Innovationspolitik beschrieben (Staab 2019).

Ungeachtet ihrer thematischen und konzeptionellen Unterschiede ist diesen Studien gemeinsam, dass sie gesellschaftliche Entwicklungen und Probleme mit Organisationen in Beziehung setzen. Eine Rückbesinnung auf das Konzept der Organisationsgesellschaft und dessen soziologisch überzeugende Neuausrichtung würde es ermöglichen, dass diese (und vergleichbare) Studien aus unterschiedlichen Teilgebieten der Soziologie sich wechselseitig zur Kenntnis nehmen und auf gewinnbringende Weise miteinander verbunden werden. Die Überzeugungskraft einer derartigen Zusammenführung verlangt jedoch, bestehende Konzeptualisierungen der Organisationsgesellschaft zu nutzen und sie so weiterzuentwickeln, dass damit aktuelle empirische Entwicklungen adäquat erfasst werden können.

Auch die Organisationsforschung kann von der hier vorgeschlagenen Forschungsperspektive profitieren. Denn dort stehen unabhängig davon, ob die historische Prägung oder die aktuelle Einbettung einzelner Organisationen betont wird, allzu oft lediglich ,organizational responses“ oder ,,interorganizational relations“ im Vordergrund. Das Problem dieser Verengung ist, dass gesellschaftliche Voraussetzungen und Folgen aus dem Blick geraten, wenn das Forschungsinteresse beispielsweise auf selektive Formen der Umweltwahrnehmung von Organisationen (im Anschluss an Weick 1995), Fragen loser Kopplung (etwa im Sinne von Meyer und Rowan 1977) oder Formen zwischenorganisatorischer Vernetzung (s. etwa Powell 1990) beschränkt ist.

In Abgrenzung zu derartigen Schwerpunkten der Organisationsforschung geht es einer organisationsgesellschaftlichen Forschungsperspektive um den Zusammenhang von Organisation und Gesellschaft. Klassischen Beiträgen zum Thema Organisationsgesellschaft ist es gelungen, gesellschaftliche Voraussetzungen und Folgen von Organisation einzubeziehen, aber verständlicherweise konnten sie aktuelle Organisationsformen noch nicht berücksichtigen. Darüber hinaus ist im Rückblick auffällig, dass es generell an einem Verständnis für die Unterschiedlichkeit vorherrschender Organisationsformen gemangelt hat. Um dies zu zeigen, legen wir im ersten Teil dieses Beitrags dar, dass in einschlägigen Klassikern die Idee der formalen, arbeitsteiligen (Groß-)Organisation dominiert, und zwar in der Organisationstheorie in Form von Bürokratien (insbesondere bei Arthur Stinchcombe 1965 und Charles Perrow 2001) und in der soziologischen Theorie als deren Weiterentwicklung zu eigensinnigen Systemen (Luhmann 2000) oder zu interessengeleiteten korporativen Akteuren (Coleman 1986). Das gemeinsame Problem dieser ansonsten grundlegend verschiedenen Beiträge aus der zweiten Hälfte des 20. Jahrhunderts ist, dass die von ihnen thematisierte Organisationsform (formal, arbeitsteilig und im Regelfall groß) heutzutage weniger dominant und alternativlos erscheint als ehedem. Und sofern wir es im 21. Jahrhundert mit anderen, neuartigen Formen der Organisation zu tun haben, ist eine Einbeziehung dieser Organisationsformen und eine Sensibilität für Organisationsunterschiede geboten. Während die Klassiker der Organisationsgesellschaft also aufgezeigt haben, dass und inwiefern das Aufkommen und die Ausbreitung von Organisation an sich einen Unterschied für die Gesellschaft gemacht hat, geht es 
in einem Update der Organisationsgesellschaft darum, aufzuzeigen, inwiefern neue Organisationsformen - und somit Organisationsunterschiede - einen Unterschied für die Gesellschaft machen.

Deshalb setzen wir uns in Abschn. 3 mit aktuellen Beiträgen auseinander, die das Aufkommen neuer Formen der Organisation hervorheben. Berücksichtigt werden aus umfangreichen Forschungsprogrammen hervorgegangene Beschreibungen, deren Ergebnisse innerhalb der Organisationssoziologie stark rezipiert worden sind und die auch darüber hinaus vielversprechende Anschlussmöglichkeiten bieten. Konkret beziehen wir uns auf das von John W. Meyer und anderen (insbesondere Bromley und Meyer 2015) beschriebene Phänomen der Hyper-Organisation, auf Varianten der von Nils Brunsson und anderen (insbesondere Ahrne und Brunsson 2019) untersuchten partiellen Organisation und auf die Plattform-Organisation, die insbesondere über Gerald F. Davis Eingang (insbesondere Davis 2016) in die Fachdiskussion gefunden haben. Wir befragen diese Beiträge danach, welche Formen der Organisation sie hervorheben und inwiefern sie gesellschaftliche Voraussetzungen und Folgen in ihre Analyse einbeziehen. Gezeigt wird, dass diese Beiträge zwar über neue Organisationsformen informieren, sie tun dies jedoch eher einseitig und suggerieren eine vollständige Ersetzung vorheriger Formen durch neue. Zudem sind sie im Hinblick auf die Einbeziehung gesellschaftlicher Voraussetzungen und Folgen unterschiedlich ausgereift. Vor diesem Hintergrund entwickeln wir das doppelte Argument, dass ein Update der Organisationsgesellschaft (erstens) organisatorische Vielfalt zu berücksichtigen und (zweitens) deren gesellschaftliche Voraussetzungen und Folgen umfassender einzubeziehen hat.

\section{Organisationsgesellschaft als klassische Forschungsperspektive}

Dass Organisationen die gesellschaftliche Entwicklung maßgeblich prägen, ist der Soziologie seit jeher bewusst. Max Weber (1972, 1982), der den Zusammenhang von gesellschaftlicher Rationalisierung und dem Organisationsprinzip der Bürokratisierung aufgezeigt hat, ist Startpunkt für die Entwicklung einer in diesem Sinne organisationsgesellschaftlichen Forschungsperspektive gewesen. Hieran anknüpfend hat es sowohl in soziologischen Theorien als auch in der Organisationssoziologie wegweisende Weiterentwicklungen gegeben, die im Folgenden skizziert werden sollen.

\subsection{Organisationssoziologische Zugänge}

Webers grundlegende These zum Zusammenhang von Organisation und Gesellschaft wurde insbesondere in Beiträgen von Arthur Stinchcombe $(1965,1986)$ aufgegriffen. Organisationsgründungen und die Zunahme an Organisationen gelten als wesentliches Merkmal gesellschaftlicher Entwicklungsniveaus. Dabei betont Stinchcombe (1965), dass es meistens andere Organisationen sind, die die Gründung einer Organisation anstoßen. So motiviert die Gründung eines erfolgreichen Unternehmens beispielsweise die Gründung konkurrierender Unternehmen, es bilden sich in expandierenden Branchen im Regelfall Zulieferer- und Abnehmer-Organisationen heraus 
und es kommt zur Entstehung weiterer Organisationen, etwa in Form von Gewerkschaften und Verbänden.

Gleichzeitig können bestehende Organisationen die gesellschaftliche Entwicklung Stinchcombe zufolge bremsen, weil von ihnen Beharrungseffekte ausgehen. Dies wird damit begründet, dass Organisationen zum Gründungszeitpunkt jeweils als funktional erachtete Prinzipien inkorporieren - zum Beispiel Arbeitsteilung im Rahmen serieller Produktionsverfahren (Ende des 19. Jahrhunderts), Forschung und Entwicklung (seit Anfang des 20. Jahrhunderts und beginnend mit der Chemischen Industrie) oder, so kann man ergänzen, Öffentlichkeitsarbeit (seit Ende des 20. Jahrhunderts an Bedeutung gewinnend). Organisationen tendieren im weiteren Verlauf ihrer Entwicklung dazu, entsprechende Merkmale zu konservieren und neuartigen Organisationsformen reserviert gegenüberzustehen. Die Ausgangsbedingungen zum Gründungszeitpunkt prägen eine Organisation demnach nachhaltig. Die hierauf bezogene These eines Imprinting von Organisationen ist insbesondere seitens der population ecology (Hannan und Freeman 1977) aufgegriffen worden und konnte durch Folgestudien empirisch belegt werden (Johnson 2007; Marquis und Tilcsik 2013).

Stinchcombes Analyse bezieht sich vornehmlich auf das 20. Jahrhundert und damit auf eine Epoche, die durch arbeitsteilige Großorganisationen dominiert ist. Der Dominanz dieser Großorganisationen werden unmittelbare sozialstrukturelle Effekte zugeschrieben, weil sie die Berufs- und Einkommensstrukturen einer Gesellschaft prägen. Diese Perspektive wird von Charles Perrow (1989, 2001) mit Bezug auf die USA des ausgehenden 19. Jahrhunderts zugespitzt. Insbesondere behandelt er dabei die Entstehung der „Fabrikbürokratie“. Aufgezeigt wird, wie sowohl im Textil- als auch im Eisenbahnwesen netzwerkbasierte Formen der Organisation durch bürokratische Großunternehmen verdrängt wurden, und zwar nicht, weil es sich um effizientere Organisationsformen gehandelt habe, sondern weil es den Eigentümern von Großunternehmen gelungen sei, ihre Macht für die Durchsetzung von für sie vorteilhaften Rahmenbedingungen zu nutzen.

Die seitdem dominierenden Großunternehmen sind sodann verantwortlich für gravierende Ungleichheiten bei der Verteilung von Erwerbseinkommen sowie für eine überaus feingliedrige Struktur organisatorischer Positionen, die für sozialen Auf- und Abstieg ausschlaggebend sind. Zudem verursachen sie soziale und ökologische Kosten, deren Externalisierung dazu führt, dass traditionelle Gruppen, wie Nachbarschaften, Familienzusammenhänge und andere Solidargemeinschaften, zunehmend überfordert sind und deshalb an Bedeutung verlieren. Hierdurch kommt es zu manifesten sozialen Problemen, derer sich Perrow zufolge zunehmend sog. Infrastrukturorganisationen annehmen. Dazu gehören Asyle, Wohlfahrtseinrichtungen oder Gefängnisse, die sodann als integrale Bestandteile der US-amerikanischen Organisationsgesellschaft des 20. Jahrhunderts ausgewiesen werden.

\subsection{Gesellschaftstheoretische Zugänge}

Gesellschaftstheoretische Zugänge zum Thema Organisationsgesellschaft setzen bereits bei der Auflösung der feudal-absolutistischen Gesellschaft Anfang des 19. Jahrhunderts und der Ausweitung von Bürgerrechten wie Wahlrecht, freie Berufswahl, Koalitions- und Meinungsfreiheit sowie dem Arbeitsvertragsrecht an (s. auch Türk 
et al. 2006). James S. Coleman (1986) argumentiert, dass sich viele dieser Bürgerrechte gegenüber dem Staat und anderen gesellschaftlichen Gruppierungen nur durchsetzen ließen, weil sich individuelle Akteure in Interessenorganisationen zusammenschlossen. Sozialtheoretischer Ausgangspunkt dieser Argumentation ist eine Handlungstheorie (Coleman 1990) und Organisationen treten zunächst als Instrumente in Erscheinung, das heißt als Mittel für verschiedene Zwecke.

Coleman rekonstruiert den hieraus hervorgehenden korporativen Akteur und den dadurch ausgelösten gesellschaftlichen Wandel zunächst anhand einer historischen Betrachtung des Rechtssystems. Die Unterscheidung zwischen natürlichen Personen und juristischen Personen ist entscheidend (s. auch Matys 2011). Einerseits wurden damit Organisationen als juristische Personen neben Individuen zu einem ebenbürtigen Strukturelement in der Gesellschaft; schließlich können juristische Personen, wie z.B. Unternehmen und Behörden, Rechte und Pflichten akkumulieren sowie für Handlungen zur Verantwortung gezogen werden (s. auch Hasse 2019). Andererseits können diese neuen korporativen Akteure über eine Machtfülle verfügen, die diejenige von natürlichen Personen weit übersteigt.

Die Entfaltungsmöglichkeiten von Individuen werden durch die Vormachtstellung von Organisationen als korporative Akteure automatisch eingeschränkt, so Colemans grundlegende These, die er unter dem Stichwort einer asymmetrischen Gesellschaft (Coleman 1986) zuspitzt. Darüber hinaus beschreibt er eine Entwicklung, die sich eigendynamisch verstärkt: Die Entstehung von Organisationen, die die Interessen einer bestimmten Gruppe vertreten, ruft die Entstehung weiterer Organisationen hervor, die Ressourcen ebenso bündeln, um so die Interessen anderer durchzusetzen (s. auch Preisendörfer 2005). Die Dominanz kapitalistischer Unternehmen und staatlicher Organisationen hat also weitere Organisationsbildungen vorangetrieben.

Auf fundamental anderen Theorieprämissen aufbauend, betrachtet Niklas Luhmann $(1975,1997)$ die Bildung formaler Organisationen als Schlüsselereignis gesellschaftlicher Entwicklung. Zwar habe es erste Formen der Organisationsbildung bereits in den klassischen Hochkulturen gegeben, doch für Luhmann ist deren massenhafte Ausbreitung an gesellschaftliche Voraussetzungen gebunden, die erst später vorliegen. Hierzu zählen Verstädterung, Verrechtlichung und Monetarisierung ebenso wie Bildung und Schriftlichkeit. Luhmanns Systemtheorie rekurriert damit im Wesentlichen auf gesellschaftliche Bedingungen, die bereits in Webers Bürokratietheorie hervorgehoben werden.

Auch seitens der Systemtheorie werden Organisationsbildung und Organisationsverdichtung als eigendynamische Prozesse beschrieben. Das heißt, es sind vor allem Organisationen, die die Gründung von weiteren Organisationen vorantreiben. Im Hinblick auf die gesellschaftlichen Folgen von Organisationsbildung ist wichtig, dass Organisationen besondere Möglichkeiten der Handlungs- und Verhaltensstabilisierung sowie der Konfliktneutralisierung zugesprochen werden (Luhmann 1964; Seidl und Mormann 2014). Entscheidend für die gesellschaftliche Entwicklung ist für ihn jedoch, dass funktionale Differenzierung auf Gesellschaftsseite Organisationsbildung sowohl voraussetzt als auch vorantreibt (Hasse und Krücken 2005).

In systemtheoretischer Lesart treiben Organisationen die funktionale Differenzierung der Gesellschaft jedoch nicht nur voran, sondern sie kompensieren zugleich auch deren Rigidität. Das heißt, sie ermöglichen strukturelle Kopplungen zum Bei- 
spiel zwischen wissenschaftlichen, wirtschaftlichen und politischen Organisationen. Ebenso wird angenommen, dass Organisationen problematische Effektübertragungen innerhalb eines gesellschaftlichen Teilsystems begrenzen können (Luhmann 2000, S. 394). Zugleich können sie genau hier scheitern, wie die Finanzkrise vor Augen geführt hat. Der Startpunkt der Finanzkrise lag demnach in der Art und Weise der Bewirtschaftung von Krediten einzelner Banken und es mangelte an organisatorischen Vorkehrungen der Unterbrechung von Interdependenzen. Entsprechend hatte eine Bankenpleite direkte Effekte auf die Liquidität anderer Banken und Unternehmen, sodass eine Kettenreaktion ausgelöst wurde (siehe z. B. Kette 2009).

\subsection{Zwischenbilanz}

Die vorgestellten Klassiker der Organisationsgesellschaftsanalyse repräsentieren verschiedene paradigmatische Positionierungen. Gleichwohl gibt es bemerkenswerte Gemeinsamkeiten. So beziehen sie sich allesamt auf Webers Bürokratietheorie. Zudem wird jeweils die Dominanz einer bestimmten Organisationsform oder ein Archetyp der Organisation angenommen - nämlich auf Arbeitsteilung basierend, formal organisiert, stabil, eindeutig von der gesellschaftlichen Umwelt abgrenzbar und im Regelfall groß. Während die Organisationsgröße für Luhmann keinen systematischen Stellenwert hat und eher implizit vorausgesetzt wird, beziehen sich sowohl Stinchcombe als auch Perrow vornehmlich auf kapitalistische Großunternehmen und schreiben vor allem ihnen sozialstrukturelle Effekte zu. Colemans Aufmerksamkeit gegenüber kapitalistischen Unternehmen und zentralen staatlichen Behörden weist ebenso auf die Relevanz der Organisationsgröße hin. Eine weitere Gemeinsamkeit der hier diskutierten Beiträge zur Analyse der Organisationsgesellschaft ist es, dass die von uns als Archetyp bezeichnete Organisationsform als so leistungsfähig und mächtig angesehen wird, dass sie andere Formen der Sozialorganisation (z. B. Familien, Gruppen oder Gemeinschaften, aber auch alternative, zum Beispiel netzwerkbasierte Organisationsformen) verdrängt hat. Und schließlich werden Unterschiede zwischen Unternehmen verschiedener Branchen und zwischen Organisationstypen wie Forschungsinstituten, Verwaltungen, Krankenhäusern etc. jeweils gering veranschlagt.

Insgesamt legt die bemerkenswerte Übereinstimmung der jeweils angenommenen Organisationsform die kritische Frage nahe, was ist, wenn das in den behandelten Klassikern der Organisationsgesellschaftsanalyse zugrunde gelegte Organisationsverständnis nicht (mehr) den sozialen Realitäten entspricht. In diesem Fall, so ist anzunehmen, wäre der Archetyp nicht mehr länger als Startpunkt für die Analyse der Gegenwartsgesellschaft überzeugend. Er bedürfte einer Aktualisierung, zu der wir im Folgenden beitragen wollen.

\section{Update: Neue Organisationsformen}

Zwar wird kaum in Frage gestellt, dass neue, teils ,unkonventionelle Formen der Organisation“ (Brès et al. 2018) an Relevanz gewinnen, doch ist ihre Konzeptualisierung bislang wenig fortgeschritten (vgl. Besio et al. 2020). Bartley et al. (2019) 
identifizieren jedoch eine wichtige, übergeordnete Veränderung in der Organisationslandschaft, die sie mit der Idee einer „concentration without centralization“ zusammenfassen. „Concentration“ ist in diesem Zusammenhang das Stichwort dafür, dass Organisationen weiterhin zentrale gesellschaftliche Akteure darstellen und insbesondere an ökonomischer Macht sogar noch gewonnen haben; ,,without centralization" verweist hingegen darauf, dass Organisationen zugleich weniger als Einheit in Erscheinung treten und auf unabgestimmte und mitunter diffuse Weise Einfluss auf die Gesellschaft nehmen.

Bei der Einbeziehung neuer Formen der Organisation geht es nicht darum, einen alles andere ersetzenden neuen Organisationstyp zu proklamieren. Vielmehr sollen die von Bartely et al. (2019) identifizierten Veränderungen für ein Update der Analyse der Organisationsgesellschaft genutzt werden. Dabei greifen wir auf einen Vorschlag von Allmendinger und Hinz (2002, S. 11) zurück, die bereits vor knapp zwei Jahrzehnten dafür plädiert haben, „Organisationsformen“ zu untersuchen, „die für die gesamtgesellschaftliche Ebene prototypisch sind“. Um hierzu einen Beitrag zu leisten, gehen wir im Folgenden auf drei aktuelle Beschreibungen neuartiger Organisationsformen ein: Plattform-Organisation (Davis 2016), partielle Organisation (Ahrne und Brunsson 2019) und Hyper-Organisation (Bromley und Meyer 2015).

Zur Illustration: Fühlen sich Universitäten zunehmend ,third missions“ jenseits von Forschung und Lehre verpflichtet oder orientieren sich Unternehmen verstärkt an abstrakten Prinzipien wie Innovativität und Diversität, dann deutet dies auf $\mathrm{Hy}$ per-Organisation hin. Hyper-Organisation entsteht dort, wo eine gegebene Organisation erstens durch gesellschaftliche Ansprüche und zweitens durch Interessen der Mitglieder derartig beeinflusst wird, dass sie sich nicht länger auf vermeintliche Kernanliegen konzentriert. Partielle Organisation bezeichnet demgegenüber Koordinationsformen jenseits klassischer Organisationen; exemplarisch sind freiwillige Übernahmen extern gesetzter Standards oder die Orientierung an regelmäßig vorgenommenen und weithin sichtbaren Ratings und Rankings. Die Organisation fairen Handels - vom Anbau bis zum Detailhandel - ist hier beispielhaft, aber auch herkömmliche B2B-Beziehungen basieren heutzutage in weiten Teilen auf partieller Organisation. Plattform-Organisationen sind schließlich dadurch charakterisiert, dass sich Organisationen zunehmend auf reine Vermittlungsaufgaben konzentrieren; prominente Beispiele sind hier Airbnb, Uber und Lieferando, die selbst weder über Personal noch über das Equipment verfügen, um die nachgefragten Dienstleistungen selbst zu erbringen.

Die genannten Beschreibungen neuartiger Organisationsformen sind vergleichsweise einfach in die Perspektive der Organisationsgesellschaft einzubeziehen. Denn sie sind nicht nur zentrale Bezugspunkte aktueller organisationssoziologischer Debatten, sondern haben die Fruchtbarkeit der Einbeziehung von Organisationsfragen auch in Forschungsfeldern jenseits der Organisationssoziologie nachgewiesen - so z. B. in der Weltgesellschaftsanalyse (Meyer und andere), zu den Themenbereichen soft regulation und new governance (Brunsson und andere) und zur Digitalisierungsforschung (Davis und andere). Wenngleich andere wichtige Beiträge, wie insbesondere die Netzwerkdebatte, teils inkludiert sind, handelt es sich bei der vorgenommenen Auswahl ausdrücklich um keine abgeschlossene Liste. Hervorzuheben ist lediglich, dass erstens nicht einfach andere Sichtweisen auf Organisation berücksich- 
tigt werden, bei denen dann berechtigterweise auch Themen wie Organisationskultur oder Organisationspraxis oder auch zwischenorganisatorische Zusammenhänge in Form von Organisationsfeldern oder -populationen einzubeziehen wären; zweitens geht es nicht darum, mitunter rasch wechselnde Best Practices oder ,managerial fads and fashions“ (Abrahamson 1991) aufzugreifen.

\subsection{Hyper-Organisation}

Mit Blick auf gesellschaftliche Entwicklungen im 21. Jahrhundert identifizieren Patricia Bromley und John W. Meyer (2015) mit der Hyper-Organisation eine Organisationsform, die sich grundlegend vom skizzierten Archetyp der Organisation unterscheidet. Hyper-Organisation zeichnet sich dadurch aus, dass Strukturen jenseits funktionaler Zusammenhänge ausgebildet werden, die entsprechend wenig mit der Erreichung eines klaren Ziels zu tun haben. Die Form der Hyper-Organisation stellt eine Weiterentwicklung zur Bürokratie dar, die gleichzeitig Elemente von freiwilligen Vereinigungen inkorporiert. Hyper-Organisationen werden als integrierte Identitäten mit umfassenden Handlungskapazitäten verstanden und treten deshalb als legitime Akteure mit weitreichenden Verantwortlichkeiten und Verpflichtungen auf. Es handelt sich um eine Organisationsform, die den Autoren zufolge in einem kontinuierlichen und anhaltenden Prozess weltweit diffundiert und sich zunehmend in verschiedenen gesellschaftlichen Sektoren (Bildung, Landwirtschaft, Gesundheit etc.) etabliert.

Hyper-Organisation ist einerseits Ausdruck einer ungebremsten Expansion von Organisation. Andererseits verweist sie auf eine Überbeanspruchung organisatorischer Strukturen für demonstrative Zwecke, die durchaus auf Kosten der Effizienz der Kernaktivitäten in einer Organisation geht - also Tätigkeiten, die gemeinhin mit einem bestimmten Organisationstypus assoziiert und von ihm erwartet werden (z.B., dass in Krankenhäusern Patienten behandelt oder in Einrichtungen des öffentlichen Nahverkehrs Personen befördert werden). Ähnlich wie Hyperventilation nicht zu verbesserter Sauerstoffversorgung führt, sind die Koordinationseffekte der Hyper-Organisation problematisch, weil immer mehr und auch solche Aufgaben übernommen werden, die mit den Kernaktivitäten einer Organisation lediglich lose verbunden sind oder diese sogar stören. Die Autoren knüpfen damit an ein überaus breites Forschungsprogramm an, das empirisch zunächst auf Organisationen des sog. institutionellen Sektors, wie z. B. Schulen, konzentriert war (Meyer und Rowan 1977) und das seit den 1990er-Jahren verstärkt auch auf Unternehmen bezogen worden ist, so z. B. im Hinblick auf die Etablierung von Diversity Management (Dobbin 2009) oder Shareholder Value-Orientierungen (Fiss und Zajac 2004).

Für eine Rekonzeptualisierung der Forschungsperspektive Organisationsgesellschaft ist relevant, dass Bromley und Meyer (2015, S. 61-92) die Ausbildung der Hyper-Organisation über gesellschaftliche Veränderungen begründen und damit Voraussetzungen dieser Organisationsform mitberücksichtigen. Ihrer Argumentation liegt ein dezidiert makrosoziologischer Ansatz zugrunde, demzufolge drei kulturelle Entwicklungen beobachtet werden können: Erstens macht die weltweite Expansion der Wissenschaft natürliche und soziale Gegenstandsbereiche kontrollierbar und eröffnet Organisationen damit immer mehr Tätigkeitsfelder. Gili S. Drori 
und John W. Meyer (2006) erklären hierzu, dass die Verwissenschaftlichung nicht nur den Glauben an eine rationale und effiziente Bearbeitung gesellschaftlicher Problemstellungen erhöht, sondern ebenso dazu führt, dass Organisationen als Akteure verstanden werden, die fähig sind, entsprechende Aktionen auszuführen. Zweitens wird die Expansion von Organisationen, diskutiert unter Schlagworten wie ,empowerment“ und ,,agency“, durch eine fortschreitende Individualisierung und eine damit einhergehende Anerkennung des Individuums als einem mit besonderen Rechten ausgestatteten Akteurs vorangetrieben, weil dies dazu legitimiert, dass sich einzelne Individuen zu zweckbestimmten Organisationen vereinen. Als dritte Voraussetzung der Hyper-Organisation wird die allgemeine Bildungsexpansion angeführt.

Die genannten Entwicklungen - Verwissenschaftlichung, Individualisierung und Bildungsexpansion - gelten als Ausdruck eines umfassenden Rationalisierungsglaubens, der praktisch jede Facette des gesellschaftlichen Lebens erfasst (Hasse und Krüger 2020, S. 20). John W. Meyer und Richard D. Scott (1983) haben dies bereits in ihren früheren Arbeiten als ein allgemeines und kulturell verankertes Organisationsprinzip betrachtet. Hyper-Organisation ist insofern der vorläufige Höhepunkt einer sich bereits länger abzeichnenden Entwicklung, die durch die Verdichtung rechtlicher oder gesellschaftlich erwarteter Regeln sowie durch technische Verfahren zur Rechenschaftspflicht (z. B. in Form von Audits, Zertifizierungen und Buchhaltung) noch beschleunigt wird.

Vor allem aber basiert Hyper-Organisation auf Professionalisierung. Diese zeigt sich im Kontext von Organisationen insbesondere durch Manager in all ihren Spezialisierungen, durch Experten (etwa zu Rechts-, Finanz- und Kommunikationsfragen) und durch technische Spezialisten, die sich Fragen der Effizienz von internen Abläufen widmen (z. B. Controller, Wirtschaftsprüfer und Prozessverantwortliche). Wenn die Effektivität ihrer jeweiligen Konzepte und Instrumente in Frage gestellt wird, reagieren diese Professionen gewöhnlich mit der Entwicklung neuer Modelle und Instrumente und steigern damit den Grad organisatorischer Durchdringung (Kühl 2009).

\subsection{Partielle Organisation}

Auch das Konzept der partiellen Organisation hebt eine vom Archetyp abweichende Organisationsform hervor. Diese ist dadurch gekennzeichnet, dass Organisation nicht ausschließlich in der Form unitärer Einheiten auftritt. Ahrne und Brunsson (2019) zufolge zeichnen sich herkömmliche Organisationen dadurch aus, dass sie zu fünf Bausteinen Entscheidungen treffen: Mitgliedschaft, Regeln, Monitoring, Sanktionen und Hierarchie. Zugleich identifizieren sie Organisation auch dort, wo lediglich Entscheidungen über einzelne oder mehrere, aber eben nicht über alle Organisationsbausteine getroffen werden. Beispiele für partielle Organisation sind Ratings, Rankings, Preise, Zertifikate oder Auszeichnungen. Sie setzen ein Monitoring voraus, basieren auf Regeln und gehen oft mit (positiven) Sanktionen einher. Sie erfordern aber weder Hierarchie noch Mitgliedschaft. Bei Bewertungen in Form von Ratings, Rankings, Preisen usw. handelt es sich um überaus gesellschaftlich folgenreiche Phänomene, weil sie zum Beispiel den Zugang von Staaten und Aktiengesellschaften zu Krediten beeinflussen. Sie haben im 21. Jahrhundert in unterschiedlichsten Gesell- 
schaftsbereichen ebenso einen enormen Bedeutungszuwachs erhalten (Stark 2020) wie Standards (Brunsson und Jacobsson 2000) und zunehmend verschiedene Gesellschaftsbereiche durchdringende Formen eines institutionell und organisatorisch abgesicherten Wettbewerbs (Arora-Jonsson et al. 2021).

Die genannten Organisationsbausteine bieten ein analytisches Instrumentarium, um verschiedene Varianten partieller Organisation zu beschreiben. So gehen Mitgliedschaftsentscheidungen in Verbrauchervereinen kaum mit Entscheidungen über Regeln, Monitoring, Sanktionen und Hierarchie einher. Auf ähnliche Weise basieren Standards zwar auf Entscheidungen über Regeln, deren Nichteinhaltung wird jedoch nicht sanktioniert; und bei Ratings sind zwar Monitoring-Systeme entscheidend, andere Bausteine (insbesondere Mitgliedschaft und Sanktionen) fehlen jedoch. Kurzum: Partielle Organisation gibt es in vielen Varianten. Sie haben jedoch gemeinsam, dass sie nicht als Akteure in Erscheinung treten (Grothe-Hammer 2019). Weder Rankings, Auszeichnungen noch Zertifikaten werden Entscheidungen zugerechnet. Wenn es um Fragen der Verantwortlichkeit geht, rücken deshalb wiederum diejenigen in den Blickpunkt, die über partielle Organisationen entscheiden. Das sind im Regelfall formale Organisationen, teils abgesichert und legitimiert durch staatliche Instanzen (Arnold und Hasse 2016).

Unabhängig davon, ob Organisation in Form einzelner oder mehrerer Organisationsbausteine auftritt, wird die Koordination der Gesellschaft dieser Argumentationslinie zufolge zunehmend partieller Organisation anvertraut. Das Update der Organisationsgesellschaft via partieller Organisation beschreibt folglich ebenso wie im Fall der Hyper-Organisation eine Organisationsexpansion. Während die HyperOrganisation jedoch Standards, Zertifizierungen oder andere Instrumente zur Rechenschaftspflicht als Mechanismen für die Expansion der formalen Organisation versteht, werden mit dem Konzept der partiellen Organisation genau diese Bausteine als verschiedene Varianten partieller Organisation betrachtet. Und während mit dem Konzept der Hyper-Organisation die These der Vergesellschaftung von Organisationen zum Ausdruck gebracht wird, rückt das Konzept der partiellen Organisation die Idee einer Organisierung der Gesellschaft auch jenseits formaler Organisationen in das Zentrum der Aufmerksamkeit.

Darüber hinaus liegen den beiden Konzepten unterschiedliche Erklärungen zugrunde. Während im Konzept der Hyper-Organisation die Ausdehnung der formalen Organisation mit kulturell-gesellschaftlichen Voraussetzungen begründet wird, resultiert bei Ahrne und Brunsson (2019) die zunehmende Dominanz von Organisationen aus einem veränderten analytischen Blick, durch den die zunehmende Bedeutung bislang vernachlässigter Organisationsphänomene fokussiert werden kann. Ihre Perspektive birgt für das Update der Organisationsgesellschaft großes Potenzial, weil damit die Rolle formaler Organisation und partieller Organisation sowie ihr Zusammenspiel in unterschiedlichsten Gesellschaftsbereichen empirisch erkundet werden können (Arnold 2020). Mit der partiellen Organisation wird folglich die organisatorische Seite der Organisationsgesellschaft aktualisiert und für Forscher greifbar gemacht. Für die gesellschaftliche Seite der Organisationsgesellschaft leistet das Konzept partieller Organisation hingegen wenig, weil die gesellschaftlichen Voraussetzungen und Folgen partieller Organisation bislang unterbelichtet sind. Pointiert 
formuliert: Ahrne und Brunnsons Vorschlag scheint ohne Gesellschaft auszukommen.

\subsection{Plattform-Organisation}

Das dritte Update bezieht sich auf das Aufkommen der Plattform-Organisation, die sich als neue Organisationsform dadurch auszeichnet, dass sie, oftmals unterstützt durch digitale Infrastrukturen, unterschiedliche Akteure miteinander verbindet. Im stabilen Kern der Plattform-Organisation finden wir gewöhnlich Internetunternehmen, d.h. formale Organisationen (Ametowobla 2020). Gemäß Gerald F. Davis (2016) hat das Aufkommen der Plattform-Organisation zur Ab- und Auflösung des klassischen Unternehmens beigetragen. Auslöser hierfür war eine gesellschaftliche Entwicklung, die er als ,Nikefizierung“ umschreibt. Illustriert am Modell des Sportartikelunternehmens Nike haben Unternehmen ihre Aktivitäten demnach zunehmend ausgelagert - und zwar nicht nur an Zulieferer in der jeweiligen Region der Produktionsstandorte, sondern weltweit.

Die durch die Nikefizierung angestoßene Erosion des klassischen Industrieunternehmens mit hoher Fertigungstiefe wird seit einigen Jahren durch eine weiterführende Entwicklung vorangetrieben, die als ,Uberisierung“ bezeichnet wird. Mit Bezug auf die wohl bekannteste Plattform-Organisation Uber, dem weltweit agierenden Vermittlungsdienst für Personenbeförderung, argumentiert Davis, dass Unternehmen ihre Aktivitäten neuerdings auf eine Vermittlungsfunktion beschränken. Das heißt, im Zuge der Uberisierung treten Unternehmen zunehmend nur noch als Intermediäre auf. Orte, Produkte und Arbeitsverhältnisse sind deshalb weniger aneinandergekoppelt (vgl. Kirchner und Beyer 2016). Die Plattform-Organisation zeichnet sich durch eine hohe Variabilität und Anpassungsfähigkeit aus, die mit einem stabilen Fokus auf die Vermittlung kombiniert wird (Baldwin und Woodard 2009; Schüßler et al. 2021). Zwar ist die Vermittlerrolle für Organisationen nichts Außergewöhnliches (vgl. Bessy und Chauvin 2013), doch ist sie im Fall der digitalen Plattform-Organisation besonders dominant.

Im Unterschied zu den anderen zwei Updates scheinen Plattform-Organisationen vornehmlich im Kontext der ,new economy“ relevant zu sein (Ametowobla 2020). Dies hängt damit zusammen, dass bisher digitale, wirtschaftsmächtige PlattformOrganisationen wie Uber, Airbnb und Deliveroo zum Untersuchungsgegenstand gemacht werden. Insofern steht mit der Plattform-Organisation zwar eine neue Form der Organisation im Fokus, diese bezieht sich jedoch primär auf Unternehmen und eben nicht auf Organisationen im Allgemeinen. Plattform-Organisationen können insofern als exemplarisch für die von Bartley et al. (2019) hervorgehobene ökonomische Machtkonzentration angesehen werden.

Ebenso werden die Ursachen für das Aufkommen der Plattformorganisation im Wirtschaftssystem verortet. Konkret macht Davis (2016) dafür die Durchsetzung der Shareholder-Value-Orientierung sowie die Finanzialisierung der Wirtschaft verantwortlich. Nicht wirtschaftliche Voraussetzungen betonen hingegen Kirchner und Beyer (2016), wenn sie argumentieren, dass erst die Digitalisierung technologische Voraussetzungen für die Ausformung von Plattform-Organisationen geschaffen habe. Dies ist konzeptionell ein wichtiger Schritt, doch könnte eine weitergehende 
Loslösung von dem wirtschaftlichen Fokus noch mehr dazu beitragen, das Update via Plattform-Organisation für eine Rekonzeptualisierung der Organisationsgesellschaft nutzen zu können. Dabei sind auch Varianten der Plattform-Organisation einzubeziehen (Schüßler et al. 2021), die aufgrund der Abwesenheit von digitalen Technologien weniger neuartig erscheinen (z. B. Auktions- und Flohmarktveranstalter) oder die außerhalb der Wirtschaft operieren (z. B. Selbsthilfe-Plattformen). Noch hat man jedoch den Eindruck, dass der Fokus der Forschung auf die auf digitale Infrastrukturen aufbauende Vermittlerrolle für Produkte und Dienstleistungen gerichtet ist (Kirchner 2019).

In analytischer Hinsicht resümiert Acquier (2018), dass die Plattform-Organisation in zweierlei Hinsicht mit dem weiter oben als Archetyp beschriebenen bürokratischen Großunternehmen bricht. Die erste Abweichung ist in organisatorischer Hinsicht folgenreich. Sie verweist darauf, dass die Plattform-Organisation die klassische Unterscheidung zwischen Markt und Hierarchie unterläuft, weil sie als Intermediär weder eindeutig der Kategorie Hierarchie noch der Kategorie Markt zugewiesen werden kann. Eine kritische Auseinandersetzung mit der dichotomen Unterscheidung zwischen Markt und Hierarchie hat allerdings bereits vor dem Auftauchen oder vor der Auseinandersetzung mit der Plattform-Organisation eingesetzt. Insbesondere Powell (1990) hat in diesem Zusammenhang gezeigt, dass Netzwerke wesentlich zur gesellschaftlichen Koordination beitragen und weder Märkten noch Hierarchien entsprechen. Merkmale der Plattform-Organisation sind demnach nicht als genuin neuartig einzustufen (s. auch Frenken und Fuenfschilling 2020), sondern vielmehr als Ausgestaltung und Fortführung eines anhaltenden Trends, nämlich weg vom Archetyp der Organisation.

Der von Acquier (2018) beschriebene zweite Bruch mit dem Großunternehmen ist für gesellschaftliche Effekte folgenreich. Demzufolge läuft die Konfliktlinie zwischen Arbeit und Kapital im Kontext der Plattform-Organisation zunehmend ins Leere, weil nicht nur Investitionen in Betriebsmittel, sondern auch die Anzahl der für sie arbeitenden Organisationsmitglieder möglichst gering gehalten werden. Insbesondere durch letztgenanntes hat die Plattform-Organisation das Potenzial, das gesamte System industrieller Beziehungen bis hin zu etablierten Formen des Wohlfahrtsstaates und dessen Finanzierung zu transformieren.

Über die Beschreibung einer bestimmten neuen Organisationsform hinaus liegt die Stärke der von Davis (2016) initiierten Perspektive nicht in der Untersuchung ihrer gesellschaftlichen (mitsamt wirtschaftlicher und technologischer) Voraussetzungen, sondern in der Ausleuchtung gesellschaftlicher Folgen wie der weitreichenden Flexibilisierung von Arbeit. Soziologische Schlüsselthemen wie soziale Ungleichheit und die Prekarisierung von Arbeit, die im Modell der Hyper-Organisation und insbesondere im Konzept der partiellen Organisation zu kurz kommen, werden mit dem Konzept der Plattform-Organisation in den Fokus der Aufmerksamkeit gerückt. Auf diese Weise liefern Analysen zur Plattform-Organisation aktuelle Hinweise darauf, dass vorherrschende Organisationsformen die Ausgestaltung sozialer Ungleichheiten mitformen (s. auch Tomaskovic-Devey und Avent-Holt 2019). Inwiefern dies auch im Hinblick auf Hyper-Organisation oder partielle Organisation anzunehmen ist und welche Zusammenhänge zwischen sozialer Ungleichheit und partieller oder Hyper-Organisation bestehen, ist demgegenüber bislang ungeklärt. 
Darüber hinaus verdeutlicht die Plattform-Organisation, dass sich auch (formale) Organisationen des 21. Jahrhunderts durch Nichtverantwortung auszeichnen können und dass dies sogar intendiert sein kann. Anders als die Hyper-Organisation, die einen Status als Akteur anstrebt und damit Verantwortlichkeit geradezu einfordert, scheint die Plattform-Organisation keinen Status als Akteur anzustreben. Sie versucht gar diesen Status zu vermeiden, indem sie sich als scheinbar neutrale Instanz inszeniert. Auf dieser Grundlage kann sie in Bereichen tätig werden, die ansonsten im Hinblick auf Status und Legitimität problematisch sein könnten. Zudem stehen Plattform-Organisationen dafür, dass, anders als die Idee der partiellen Organisation suggeriert, Verantwortlichkeitsexternalisierung nicht unbedingt mit der Auflösung von (formaler) Organisation einhergehen muss. Insofern verweist die Diskussion über die Plattform-Organisation im Hinblick auf aktuelle Fragen der Verantwortlichkeitsübernahme auf einen dritten Weg zwischen partieller Organisation und HyperOrganisation.

Dieser Weg ist allerdings noch schwach ausgeleuchtet. Denn während für den Fall der Hyper-Organisation und der partiellen Organisation empirische Evidenzen für entsprechende Entwicklungen vorliegen, ist im Fall einer vermeintlichen Uberisierung einzugestehen, dass neu gegründete Organisationen des 21. Jahrhunderts nicht unbedingt Plattform-Organisationen sind. Die Plattform-Organisation scheint nach wie vor eine Ausnahmeerscheinung zu sein, die jedoch aufgrund ihrer Andersartigkeit und dem offensichtlichen Bruch mit klassischen Unternehmen für viel mediale, gesellschaftspolitische und auch wissenschaftliche Aufmerksamkeit sorgt. Ihre Bedeutung wird jedoch möglicherweise überschätzt.

Gegen die Vorstellung einer neuen Dominanz der Plattform-Organisation spricht zudem, dass diese neue Organisationsform reguläre, formale Organisationen unabdingbar voraussetzt (z. B. Brandl 2020). Weil die Plattform-Organisation Intermediär ist und unterschiedliche Einheiten miteinander in Verbindung setzt, braucht sie diese Parteien, zwischen denen sie vermitteln kann. Das Beispiel Uber zeigt, dass zwar auch Individuen diese zu vermittelnden Einheiten sein können, doch in vielen Fällen handelt es sich (zumindest auf Seiten der Anbieter) um Unternehmen im klassischen Sinne, oftmals sogar kleinerer oder mittlerer Größe. Bemerkenswerterweise sind dies oft gewöhnliche Einzelhändler, Restaurants, Pensionen oder Landwirtschaftshöfe und somit Organisationsformen, die man in Anlehnung an Stinchcombe (1965) als vormodern bezeichnen könnte und die durch die Plattform-Organisation eine Renaissance erfahren könnten. Mit Blick auf Erscheinungsformen der Organisationsgesellschaft des 21. Jahrhunderts gilt es deshalb, die Organisationsvielfalt mit ihren Unterschieden anzuerkennen, ohne punktuelle oder sektorale Veränderungen vorschnell zu Trends zu überhöhen.

\section{Diskussion}

Klassischen Konzeptualisierungen zufolge sind Organisationen Entitäten, die sich durch Arbeitsteilung, Formalisierung, Grenzziehung und, explizit hervorgehoben oder implizit vorausgesetzt, Größe auszeichnen. Diesem Verständnis zufolge strahlen Organisationen ein hohes Maß an Stabilität aus. Die neuen Organisationsformen 
Hyper-Organisation, partielle Organisation und Plattform-Organisation führen uns jedoch vor Augen, dass diese archetypische Organisationsvorstellung die Wirklichkeit des 21. Jahrhunderts nicht unbedingt widerspiegelt und entsprechende Entwicklungen, zumindest teilweise, in andere Richtungen deuten. Soziologen, die sich mit aktuellen Organisationsformen auseinandersetzen, sind demnach gut beraten, zu berücksichtigen, dass Theorien und Modelle, die am Archetyp orientiert sind, nicht zwingend treffend sind. Es geht folglich nicht nur darum, gesellschaftliche Voraussetzungen und Folgen von Organisation im Blick zu behalten, sondern auch darum, den Entwicklungen auf der organisatorischen Seite Rechnung zu tragen. Deshalb haben wir, im Anschluss an eine Skizze klassischer Beiträge zum Thema Organisationsgesellschaft, Vorschläge zur Beschreibung neuer Organisationsformen erstmals zusammengeführt und miteinander verglichen.

Gemeinsam haben Hyper-Organisation, partielle Organisation und Plattform-Organisation, dass sie sich allesamt vom archetypischen Organisationsverständnis abgrenzen. Allerdings unterscheiden sich diese drei neuen Organisationsformen auch grundlegend voneinander. Während die Hyper-Organisation durch ein hohes Maß an Responsivität gegenüber gesellschaftlichen Erwartungen gekennzeichnet ist, halten dem Modell der partiellen Organisation zufolge einzelne Organisationsbausteine Einzug in unterschiedliche Bereiche. Die Plattform-Organisation kombiniert demgegenüber Merkmale formaler und partieller Organisation, wodurch Organisationsgrenzen verschwimmen.

Beschreibungen zur Hyper-Organisation, partiellen Organisation und PlattformOrganisation sind bereits intensiv erforschte Startpunkte, um die sich wandelnde organisatorische Seite der Organisationsgesellschaft zu erfassen. Allerdings eröffnen die einzelnen Beschreibungen nur einen selektiven Blick auf Organisationsveränderungen. Ein erstes Problem ist, dass (fälschlicherweise) vermutet werden könnte, die heutige Gesellschaft sei entweder durch Hyper-Organisation oder partielle Organisation oder Plattform-Organisation dominiert. Ein zweites Problem ist, dass jedes dieser drei Updates die (falsche) Schlussfolgerung provoziert, die von ihnen hervorgehobene Organisationsform könnte ihren Vorgänger, also den Archetyp, nicht bloß ergänzen, sondern ersetzen.

Erst in der Zusammenschau und unter Einbeziehung des Archetyps ist es möglich, die sich wandelnde organisatorische Seite der Organisationsgesellschaft zu erfassen. Dies hat nicht zuletzt forschungsstrategische Konsequenzen, denn um empirische Hinweise darauf zusammenzutragen, dass Organisationsunterschiede einen gesellschaftlichen Unterschied machen, sollte eine aktualisierte Perspektive der Organisationsgesellschaft den Wandel von Organisationsformen ebenso wie deren Varietät zu einem gegebenen Zeitpunkt anerkennen. Ohne an dieser Stelle eine systematische Auseinandersetzung mit methodischen Implikationen leisten zu können, erfordert dies vergleichende Forschungsstrategien, so wie sie in der Organisationsforschung bereits zur Diskussion gestellt worden sind (King, Felin und Whetten 2009). Dabei geht es nicht nur um synchrone Vergleiche, z. B. zwischen Ländern und Sektoren oder anders begründete Organisationsunterschiede, sondern auch um diachrone Vergleiche, mit denen Veränderungen im Zeitverlauf erfasst werden können. Methodische Anknüpfungspunkte könnte hier die vergleichende Institutionenanalyse bieten, bei der auch historische Perspektiven (Thelen 2010) und qualitative Daten 
unter Berücksichtigung verschiedener Untersuchungsebenen (Kogut 2010) einbezogen werden.

Konzeptionell ist demgegenüber entscheidend, dass ein Update der Organisationsgesellschaft gesellschaftliche Voraussetzungen und Folgen veränderter Organisationsformen einzubeziehen hat. Zunächst zu den gesellschaftlichen Voraussetzungen, deren Untersuchung eine Auseinandersetzung mit polit-ökonomischen, sozialstrukturellen und kulturellen Kontexten bedingt. Im Fall der Plattform-Organisation wurden bislang vorwiegend neue Möglichkeiten kapitalistischen Gewinnstrebens thematisiert. Hierzu zählen neue Informations- und Kommunikationstechnologien, neue Techniken des Vergleichens als Grundlage für Investitionsentscheidungen sowie ideologische Entwicklungen. Inwiefern anderweitige gesellschaftliche Voraussetzungen, wie Bildungsexpansion und Verwissenschaftlichung oder Ökologisierung die Ausbreitung der Plattform-Organisation beeinflussen und welche Legitimationsgrundlagen dabei eine Rolle spielen, sind in diesem Forschungszusammenhang demgegenüber (noch) keine zentralen Fragestellungen.

Für die partielle Organisation scheint die wichtigste gesellschaftliche Voraussetzung ein erhöhter gesellschaftlicher Koordinationsbedarf zu sein. Ursachen hierfür sind bislang im Detail nicht nachgezeichnet worden, können jedoch im Zusammenspiel von Globalisierung und Deregulierung vermutet werden (Sahlin und Wedlin 2008). Ebenso fehlt eine Perspektive, die danach fragt, was die Angebotsseite motiviert, beispielsweise Standards zu setzen, Rankings zu erstellen oder Audits durchzuführen - also partielle Organisation überhaupt zu betreiben. Eine derartige Perspektive wäre einzubeziehen, weil Verweise auf gesellschaftliche Funktionen als Erklärung für die Entstehung und Ausbreitung generell nicht ausreichen.

Im Fall der Hyper-Organisation dominieren demgegenüber bislang sozialstrukturelle und kulturtheoretische Erklärungen für ihre weltweite und Sektoren übergreifende Ausbreitung. Damit sind wichtige gesellschaftliche Voraussetzungen der Hyper-Organisation gut beleuchtet. Es bleibt jedoch unbeachtet, inwiefern technologische Entwicklungen das Aufkommen und die Ausbreitung der Hyper-Organisation beeinflussen. So wird wichtigen Trends wie der Digitalisierung der Gesellschaft keine gesonderte Aufmerksamkeit zuteil und die ansonsten breit diskutierten Rahmenbedingungen in Form von Materialität und Infrastruktur werden bestenfalls sporadisch in Rechnung gestellt.

In Bezug auf gesellschaftliche Folgen neuer Organisationsformen lässt sich allgemein bestätigen, dass bis heute ,der Einfluss von Organisationen auf ihre Umwelt, [...] selten untersucht" (Allmendinger und Hinz 2002, S. 18) worden ist. Zur Schließung dieser Forschungslücke bieten die neuen Organisationsformen vielversprechende Anschlussmöglichkeiten, mehr aber noch nicht. Für den Fall der HyperOrganisation werden beispielsweise makrosoziologische Homogenisierungstendenzen sowie Formen loser Kopplung auf Organisationsebene betont. Dies legt die Frage nahe, welche gesellschaftlichen Rückwirkungen damit einhergehen. So könnten an Hyper-Organisationen adressierte Neuerungserwartungen in Richtung Innovation oder Kreativität dazu führen, dass immer mehr Probleme oder Lösungsvorschläge um Aufmerksamkeit konkurrieren, Möglichkeiten der Umsetzung aber reduziert sind, weil die „Halbwertszeiten“ von Problemdefinitionen abnehmen (Hasse und Nyfeler 2019). 
Für die verschiedenen Varianten der partiellen Organisation ist gezeigt worden, dass diese die Grundlage für die Konstruktion neuer Märkte und Wettbewerbsfelder sind (Ahrne et al. 2015; Arora-Jonsson et al. 2020). Wie aber verändern sich Verantwortungszuschreibungen durch eine partielle Organisation? Während sich Organisationen im 20. Jahrhundert zu gesellschaftlich akzeptierten Akteuren entwickelt haben, scheint dies im 21. Jahrhundert nicht mehr auf alle Organisationsformen gleichermaßen zuzutreffen. Inwiefern sich dieser organisatorische Wandel auf die Konstruktion von Verantwortungsverhältnissen auswirken wird, ist angesichts der großen gesellschaftlichen Herausforderungen (z. B. Klimawandel, soziale Ungleichheit und Ausbeutung) eine der zentralen Fragen, die es in die Analyse der Organisationsgesellschaft einzubeziehen gilt.

Auch für den Fall der Plattform-Organisation scheinen Fragen im Hinblick auf die Konstruktion und Zuschreibung von Verantwortung zentral zu sein. Das gilt insbesondere im Kontext neuer Formen der Erwerbstätigkeit. Darüber hinaus ist die Frage naheliegend, inwiefern die Plattform-Organisation zu einer Renaissance von kleinen und mittleren Unternehmen und Startups führt, weil es oftmals deren Produkte und Dienstleistungen sind, die angeboten und vermittelt werden - und inwiefern dies eine ideologische Rehabilitation derjenigen Milieus bewirkt, die von Pierre Bourdieu (1982), John Goldthorpe (1980) und vielen anderen noch geringschätzig als Kleinbürgertum bezeichnet wurden und über deren schwindende Bedeutung lange Zeit Einigkeit zu herrschen schien.

Die hier skizzierten Themen- und Fragestellungen zu gesellschaftlichen Rahmenbedingungen neuer Organisationsformen sind Auftakt für Fragen, deren Bearbeitung ein Update der Organisationsgesellschaft ermöglicht. Für die Weiterführung und Konsolidierung dieser Perspektive wird es entscheidend sein, inwiefern es gelingt, theoretische Mittel zu koordinieren und Forschungsstrategien zu identifizieren, die die Rolle der Organisation in der Gesellschaft zu greifen vermögen. Das Vorhaben, die Organisationsgesellschaft im 21. Jahrhundert mit ihren verschiedenen Organisationsformen zu untersuchen, ist entsprechend ambitioniert. Es birgt jedoch das Potenzial, die Soziologie entscheidend zu bereichern. Dies setzt jedoch voraus, das eingangs als Elefant im Raum beschriebene Problem offen zu adressieren, das heißt, die organisatorische Durchdringung der Gesellschaft und die gesellschaftliche Relevanz von Organisationen sichtbar zu machen und ein Verständnis dafür zu entwickeln, welchen gesellschaftlichen Unterschied jeweilige Formen der Organisation - also Organisationsunterschiede - machen.

Funding Open access funding provided by University of Luzern

Open Access Dieser Artikel wird unter der Creative Commons Namensnennung 4.0 International Lizenz veröffentlicht, welche die Nutzung, Vervielfältigung, Bearbeitung, Verbreitung und Wiedergabe in jeglichem Medium und Format erlaubt, sofern Sie den/die ursprünglichen Autor(en) und die Quelle ordnungsgemäß nennen, einen Link zur Creative Commons Lizenz beifügen und angeben, ob Änderungen vorgenommen wurden.

Die in diesem Artikel enthaltenen Bilder und sonstiges Drittmaterial unterliegen ebenfalls der genannten Creative Commons Lizenz, sofern sich aus der Abbildungslegende nichts anderes ergibt. Sofern das betreffende Material nicht unter der genannten Creative Commons Lizenz steht und die betreffende Handlung nicht nach gesetzlichen Vorschriften erlaubt ist, ist für die oben aufgeführten Weiterverwendungen des Materials die Einwilligung des jeweiligen Rechteinhabers einzuholen. 
Weitere Details zur Lizenz entnehmen Sie bitte der Lizenzinformation auf http://creativecommons.org/ licenses/by/4.0/deed.de.

\section{Literatur}

Abrahamson, Eric. 1991. Managerial Fads and Fashions: The Diffusion and Rejection of Innovations. Academy of Management Review 16:586-612.

Acquier, Aurélien. 2018. Uberization Meets Organizational Theory: Platform Capitalism and the Rebirth of the Putting-Out System. In The Cambridge Handbook of the Law of the Sharing Economy, Cambridge Law Handbooks, Hrsg. Nestor M. Davidson, Michèle Finck, und Nestor M. Davidson, 13-26. Cambridge: Cambridge University Press.

Ahrne, Göran, und Nils Brunsson. 2019. Organization Outside Organizations: The Abundance of Partial Organization in Social Life. Cambridge: Cambridge University Press.

Ahrne, Göran, Patrik Aspers und Nils Brunsson. 2015. The Organization of Markets. Organization Studies $36: 7-27$.

Allmendinger, Jutta, und Thomas Hinz. 2002. Perspektiven der Organisationssoziologie. In Organisationssoziologie. Sonderband 42 der Kölner Zeitschrift für Soziologie und Sozialpsychologie, Hrsg. Jutta Allmendinger und Thomas Hinz, 42:9-28. Opladen: Westdeutscher Verlag.

Ametowobla, Dzifa. 2020. Die Plattformarchitektur als Strukturmuster. Ein Plattformbegriff für die Soziologische Debatte. TUTS-Working Papers 3.

Arnold, Nadine, und Raimund Hasse. 2016. Die Organisation von Wertzuschreibungen: Zur Bedeutung von Drittparteien für die Signalisierung moralischer Qualitäten in Märkten. Berliner Journal für Soziologie 26:329-351.

Arnold, Nadine. 2020. Accountability in Transnational Governance. The Partial Organization of Voluntary Sustainability Standards in Long-Term Account-Giving. Regulation \& Governance, online first.

Arora-Jonsson, Stefan, Nils Brunsson und Raimund Hasse. 2020. Where does competition come from? Organization Theory 1:1-24.

Arora-Jonsson, Stefan, Nils Brunsson und Raimund Hasse. 2021. The origins of competition-institution and organization. In Competition. What it is and why it happens, Hrsg. Stefan Arora-Jonsson, Nils Brunsson, Raimund Hasse und Katarina Lagerström, 61-76. Oxford: Oxford University Press.

Baldwin, Carliss Y., und C. Jason Woodard. 2009. The Architecture of Platforms: A Unified View. In Platforms, Markets and Innovation, Hrsg. Annabelle Gawer, 19-44. Cheltenham. Edward Elgar.

Bartley, Tim, Matthew Soener und Carl Gershenson. 2019. Power at a Distance: Organizational Power across Boundaries. Sociology Compass 13:e12737.

Besio, Cristina, Paul du Gay und Kathia Serrano Velarde. 2020. Disappearing Organization? Reshaping the Sociology of Organizations. Current Sociology 68:411-418.

Bessy, Christian, und Pierre-Marie Chauvin. 2013. The Power of Market Intermediaries: From Information to Valuation Processes. Valuation Studies 1:83-117.

Borchgrevink, Kaja. 2017. NGOization of Islamic Charity: Claiming Legitimacy in Changing Institutional Contexts. VOLUNTAS: International Journal of Voluntary and Nonprofit Organizations 31:1049-1061.

Bourdieu, Pierre. 1982. Die feinen Unterschiede. Kritik der gesellschaftlichen Urteilskraft. Frankfurt am Main: Suhrkamp.

Brandl, Barbara. 2020. Ist Blockchain das Ende der Banken? Zur Bedeutung von Schulden und Banken in kapitalistischen Ökonomien. Kölner Zeitschrift für Soziologie und Sozialpsychologie 72:543-565.

Bromley, Patricia, und John W. Meyer. 2015. Hyper-Organization: Global Organizational Expansion. Oxford: Oxford University Press.

Brunsson, Nils, und Bengt Jacobsson. Hrsg. 2000. A World of Standards. Oxford: Oxford University Press.

Brès, Luc, Emmanuel Raufflet und Johnny Boghossian. 2018. Pluralism in Organizations. Learning from Unconventional Forms of Organization. International Journal of Management Review 30:364-386.

Coleman, James S. 1986. Die asymmetrische Gesellschaft. Vom Aufwachsen mit unpersönlichen Systemen. Weinheim: Beltz Verlag.

Coleman, James S. 1990. Foundations of Social Theory. Cambridge, London: Harvard University Press.

Dahrendorf, Ralf. 1957. Soziale Klassen und Klassenkonflikt in der industriellen Gesellschaft. Stuttgart: Enke.

Davis, Gerald F. 2016. The Vanishing American Corporation: Navigating the Hazards of a New Economy. San Francisco: Berrett-Koehler.

Dobbin, Frank. 2009. Inventing Equal Opportunity. Princeton, NJ: Princeton University Press. 
Dolata, Ulrich. 2015. Volatile Monopole. Konzentration, Konkurrenz und Innovationsstrategien der Internetkonzerne. Berliner Journal für Soziologie 24:505-529.

Drori, Gili S., und John W. Meyer 2006. Global Scientization: An Environment for Expanded Organization. In Globalization and Organization, Hrsg. Gili S. Drori, John W. Meyer und Hokyu Hwang, 50-68. Oxford: Oxford University Press.

Duncan, Jessica, und Stefano Pascucci. 2017. Mapping the Organisational Forms of Networks of Alternative Food Networks: Implications for Transition. Sociologia Ruralis 57:316-339.

Fiss, Peer C., und Edward Zajac. 2004. The Diffusion of Ideas over Contested Terrain: The (Non)adoption of Shareholder Value Orientation among German Firms. Administrative Science Quarterly 49:501-534.

Fligstein, Nei, und Taek-Jin Shin. 2007. Shareholder Value and the Transformation of the U.S. Economy, 1984-2001. Sociological Forum 22:399-424.

Foucault, Michel. 1996. Die Geburt der Klinik: eine Archäologie des ärztlichen Blicks. Frankfurt am Main: Fischer-Taschenbuch-Verlag.

Frenken, Koen, und Lea Fuenfschilling. 2020. The Rise of Online Platforms and the Triumph of the Corporation. Sociologica 14:101-113.

Gabriel, Karl. 1979. Analysen der Organisationsgesellschaft. Ein kritischer Vergleich der Gesellschaftstheorien Max Webers, Niklas Luhmanns und der phänomenologischen Soziologie. Frankfurt am Main: Campus.

Goffman, Erving. 1995. Asyle - Über die soziale Situation psychiatrischer Patienten und anderer Insassen. Frankfurt am Main: Suhrkamp.

Goldthorpe, John. 1980. Social Mobility and Class Structure in Modern Britain. Oxford: Clarendon.

Groot Kormelinck, Annemarie, Jos Bijman und Jaques Trienekens. 2019. Characterizing Producer Organizations: The Case of Organic Versus Conventional Vegetables in Uruguay. Journal of Rural Studies 69:65-75

Grothe-Hammer, Michael. 2019. Organization Without Actorhood: Exploring a Neglected Phenomenon. European Management Journal 37:325-338.

Hannan, Michael T., und John H. Freeman. 1977. The Population Ecology of Organizations. American Journal of Sociology 82:929-964.

Hasse, Raimund. 2003. Wohlfahrtspolitik und Globalisierung. Zur Diffusion der World Polity durch Organisationswandel und Wettbewerbsorientierung. Opladen: Leske + Budrich.

Hasse, Raimund. 2019. What Difference Does it Make? An Institutional Perspective on Actors and Types thereof. Research in the Sociology of Organizations 58:23-41.

Hasse, Raimund, und Anne K. Krüger. 2020. Außenbezüge, Binnendifferenzen und neue Herausforderungen des Neo-Institutionalismus. Eine Übersicht. In Neo-Institutionalismus. Kritik und Weiterentwicklung eines sozialwissenschaftlichen Forschungsprogramms, Hrsg. Raimund Hasse und Anne K. Krüger, 9-34. Bielefeld: Transcript.

Hasse, Raimund, und Judith Nyfeler. 2019. Alles nur Mode? Organisation und Sprache in den Creative Industries. Zeitschrift für Soziologie 48:401-417.

Hasse, Raimund, und Georg Krücken. 2005. Der Stellenwert von Organisationen in Theorien der Weltgesellschaft. Zeitschrift für Soziologie, Sonderband Weltgesellschaft:186-204.

Jäger, Wieland, und Uwe Schimank. Hrsg. 2005. Facetten der Organisationsgesellschaft. Opladen: Verlag Sozialwissenschaften.

Johnson, Victoria. 2007. What Is Organizational Imprinting? Cultural Entrepreneurship in the Founding of the Paris Opera. American Journal of Sociology 113:97-127.

Jung, Jiwook, und Frank Dobbin. 2014. Finance and Institutional Investors. In The Oxford Handbook of the Sociology of Finance, Hrsg. Karin Knorr Cetina und Alex Preda, 52-74. Oxford: Cambridge University Press.

Kette, Sven 2009. Bankenregulierung als Cognitive Governance. Eine Studie zur gesellschaftlichen Verarbeitung von Komplexität und Nichtwissen. Wiesbaden: VS Verlag.

King, Brayden, Teppo Felin und David A. Whetten. 2009. Studying Differences between Organizations: Comparative Approaches to Organizational Research. Research in the Sociology of Organizations 26:91-116.

Kirchner, Stefan. 2019. Arbeiten in der Plattformökonomie: Grundlagen und Grenzen von „Cloudwork“ und „Gigwork“. Kölner Zeitschrift für Soziologie und Sozialpsychologie 71:3-25.

Kirchner, Stefan, und Jürgen Beyer. 2016. Die Plattformlogik als digitale Marktordnung. Zeitschrift für Soziologie 45:324-339.

Kogut, Bruce. 2010. Qualitative Comparative Analysis of Social Science Data. In The Oxford Handbook of Comparative Institutional Analysis, Hrsg. Glenn Morgan, John L. Campbell, Colin Crouch, Ove Kaj Pedersen und Richard Whitley, 139-179. New York: Oxford University Press. 
Kühl, Stefan. 2009. Capacity Development as the Model for Development Aid Organizations. Development and Change 40:551-577.

Kühl, Stefan. 2015. Gesellschaft der Organisationen, organisierte Gesellschaft, Organisationsgesellschaft. In Zur Zukunft der Organisationssoziologie. Hrsg. Maja Apelt und Uwe Wilkesmann, 73-91. Wiesbaden: Springer.

Luhmann, Niklas. 1964. Funktionen und Folgen formaler Organisation. Berlin: Duncker \& Humblot.

Luhmann, Niklas. 1975. Soziologische Aufklärung 2: Aufsätze zur Theorie der Gesellschaft. Opladen: Westdeutscher Verlag.

Luhmann, Niklas. 1997. Die Gesellschaft der Gesellschaft. Frankfurt am Main: Suhrkamp.

Luhmann, Niklas. 2000. Organisation und Entscheidung. Opladen: Westdeutscher Verlag.

Marquis, Christopher, und András Tilcsik. 2013. Imprinting: Toward a Multilevel Theory. The Academy of Management Annals 7:193-243.

Matys, Thomas. 2011. Legal Persons - „Kämpfe“ und die organisationale Form. Wiesbaden: VS Verlag für Sozialwissenschaften.

McQuarrie, Michael, und Nicole P. Marwell. 2009. The Missing Organizational Dimension in Urban Sociology. City \& Community 8:247-268.

Meyer, John W., und Rowan, Brian. 1977. Institutionalized Organizations: Formal Stucture as Myth and Ceremony. American Journal of Sociology 83:340-363.

Meyer, John W., und W. Richard Scott. 1983. Organizational Environments: Ritual and Rationality. London: Sage.

Paternotte, David. 2016. The NGOization of LGBT Activism: ILGA-Europe and the Treaty of Amsterdam. Social Movement Studies 15:388-402.

Perrow, Charles. 1989. Eine Gesellschaft von Organisationen. Journal für Sozialforschung 29:3-19.

Perrow, Charles. 2001. Organizing America: Wealth, Power, and the Origins of Corporate Capitalism. Princeton, NJ: Princeton University Press.

Powell, Walter W. 1990. Neither Market nor Hierarchy: Network Forms of Organization. Research in Organizational Behavior 12:295-336.

Preisendörfer, Peter. 2005. Organisationssoziologie. Grundlagen, Theorien und Problemstellungen. Wiesbaden: VS Verlag für Sozialwissenschaft.

Sahlin, Kerstin, und Linda Wedlin. 2008. Circulating Ideas: Imitation, Translation and Editing. In The SAGE Handbook of Organizational Institutionalism, Hrsg. Royston Greenwood, Christine Oliver, Roy Suddaby und Kerstin Sahlin, 218-242. London: Sage.

Schimank, Uwe. 2010. Die funktional differenzierte kapitalistische Gesellschaft als Organisationsgesellschaft. Eine theoretische Skizze. In Die Ökonomie der Organisation - die Organisation der Ökonomie, Hrsg. Martin Endreß und Thomas Matys, 33-61. Wiesbaden: VS Verlag für Sozialwissenschaft.

Schüßler, Elke, Will Attwood-Charles, Stefan Kirchner und Juliet B. Schor. 2021. Between mutuality autonomy and domination: rethinking digital platforms as contested relational structures. Socio-Economic Review. https://doi.org/10.1093/ser/mwab038.

Seidl, David, und Hannah Mormann. 2014. Niklas Luhmann as Organization Theorist. In The Oxford Handbook of Sociology, Social Theory, and Organization Studies: Contemporary Currents, Hrsg. Paul Adler, Paul du Gay, Glenn Morgan und Mike Reed, 125-157. Oxford: Oxford University Press.

Staab, Philipp. 2019. Digitaler Kapitalismus. Markt und Herrschaft in der Ökonomie der Unknappheit. Frankfurt am Main: Suhrkamp.

Stark, David. Hrsg. 2020. The Perfomance Complex. Oxford: Oxford University Press.

Stinchcombe, Arthur L. 1965. Social Structure and Organizations. In Handbook of Organizations. Hrsg. James. G. March, 153-193. Chicago: Rand McNally.

Stinchcombe, Arthur L. 1986. Stratification and Organizations: Selected Papers. England: Cambridge University Press.

Thelen, Kathleen. 2010. Beyond Comparative Statics: Historical Institutional Approaches to Stability and Change in the Political Economy of Labor. In The Oxford Handbook of Comparative Institutional Analysis, Hrsg. Glenn Morgan, John L. Campbell, Colin Crouch, Ove Kaj Pedersen und Richard Whitley, 41-62. New York: Oxford University Press.

Tomaskovic-Devey, Donald, und Dustin Avent-Holt. 2019. Relational Inequalities: An Organizational Approach. Oxford: Oxford University Press.

Türk, Klaus, Thomas Lemke und Michael Bruch. Hrsg. 2006. Organisation in der modernen Gesellschaft. Eine historische Einführung. Wiesbaden: Springer VS.

Weber, Max. 1972. Wirtschaft und Gesellschaft. Grundriss der verstehenden Soziologie. 5. Aufl., Tübingen: Mohr.

Weber, Max. 1982. Die protestantische Ethik und der Geist des Kapitalismus. 4. Aufl., Tübingen: Mohr. 
Weick, Karl E. 1995. Sensemaking in organizations. Foundations for organizational science. London: Sage.

Whitley, Richard 1984. The Intellectual and Social Organization of the Sciences. Oxford: Oxford University Press.

Nadine Arnold Dr. phil., Oberassistentin am Soziologischen Seminar der Universität Luzern. Aktuelle Publikationen: Serving magically perfect fruit globally: local nesting in translating multiple standards. Organization Studies 42, 2021; Accountability in transnational governance: The partial organization of voluntary sustainability standards in long-term account-giving. Regulation \& Governance, online first, 2020; Wenn Food Waste sichtbar wird: Zur Organisation und Bewertung von Lebensmittelabfällen. Bielefeld 2021 (als Hrsg.).

Raimund Hasse Dr. rer. soc., Professor für Soziologie: Organisation und Wissen an der Universität Luzern. Aktuelle Publikationen: Alles nur Mode? Organisation und Sprache in den Creative Industries. Zeitschrift für Soziologie 48, 2019 (mit J. Nyfeler); Neo-Institutionalismus: Kritik und Weiterentwicklung. Bielefeld 2020, (als Hrsg., mit A. Krüger); Competition. What it is and why it happens. Oxford University Press (ed. 2021, with Stefan Arora-Jonsson, Nils Brunsson und Katharina Lagerström).

Hannah Mormann Dr. phil., Oberassistentin am Soziologischen Seminar der Universität Luzern. Aktuelle Publikationen: Diversity-Trainings und das Prinzip der Individualisierung. Eine neo-institutionalistische Analyse. ZDfm - Zeitschrift für Diversitätsforschung und -management 5, 2020; Zwischen Heuchelei und Heldentum. Zur Rolle von Organisationen in der sozial-ökologischen Transformation der Gesellschaft. Soziologie und Nachhaltigkeit 5, 2019 (mit N. Arnold). 